\title{
Collagen Content and Distribution in the Normal and Transplanted Human Heart: A Postmortem Quantitative Light Microscopic Analysis
}

\author{
R. I. van Suylen, MD,* E. E. C. van Bekkum* H. Boersma, MSc ${ }^{\dagger}$ L. B. de Kok, MSc, ${ }^{\ddagger}$ \\ A. H. M. M. Balk, MD, PhD,** E. Bos, MD, PhD, ${ }^{\dagger \dagger}$ and F. T. Bosman, MD, PhD* \\ From the Departments of *Pathology, ${ }^{* *}$ Cardiology, ${ }^{\dagger}$ Clinical Epidemiology, and ${ }^{\dagger+}$ Thoracic Surgery, Thorax Centre, \\ Erasmus University, Rotterdam; and ${ }^{\ddagger}$ Department of Pathology, Diagnostic Centre "SSDZ," Delfi, Netherlands
}

The myocardium consists of a muscular, a vascular, and an interstitial compartment. Collagen is the major structural component of the myocardial interstitium and maintains the structural integrity of the heart. In pathological conditions (ischemic heart disease, cardiomyopathy, hypertension, valvular disease, etc.) excessive collagen production may contribute

Manuscript received January 3, 1995; accepted July 25, 1995

Address for reprints: R. J. van Suylen. Department of Pathology, academisch ziekenhuis Maastricht, P. Debyelaan 25, P.O. Box 5800, 6202 AZ Maastricht, Netherlands: telephone: 3143874612 , fax: 3143876613. to increased myocardial stiffness, resulting in impaired cardiac function (1-11). In the transplanted heart also interstitial fibrosis has been observed, and this could potentially have an adverse effect on long-term function of the cardiac allograft $(12-15)$

The major long-term changes that occur in the transplanted heart and which may have adverse effects on long term cardiac function are arteriosclerosis, interstitial fibrosis and myocy te hypertrophy. Some studies have raised doubts about the presence of increased interstitial fibrosis in the transplanted heart and its contribution to cardiac dysfunction $(16,17)$. Endomyo- 
cardial biopsies offer the opportunity to study the myocardial interstitium in the context of clinical parameters of myocardial function. For that purpose, however, endomyocardial biopsies should reliably reflect the composition of the entire myocardium.

Only a few reports have addressed the problem of whether or not small endomyocardial biopsies are representative for the entire myocardium $(18,19)$. Baandrup et al. have proposed that at least five endomyocardial biopsies have to be obtained in order to assess reliably the collagen content in the interventricular septum (20). This issue is important because it determines the potential value of the right ventricular endomyocardial biopsy in the analysis of the left ventricular function. In the present study we determined the collagen content in the subendocardial region and central parts of the right and left ventricular free wall and interventricular septum of 16 normal human hearts and 30 hearts of patients who died. with different intervals, after heart transplantation.

First, we determined whether the collagen content in the subendocardial region of the right side of the interventricular septum (site of right ventricular endomyocardial biopsy). in normal and transplanted human hearts, is representative for the entire myocardium.

Second, in the transplanted hearts we determined whethet or not the mean collagen content of the right and left ventricle and the interventricular septum is altered along with the posttransplantation survival time

Third, we attempted to identify transplantation related fac-
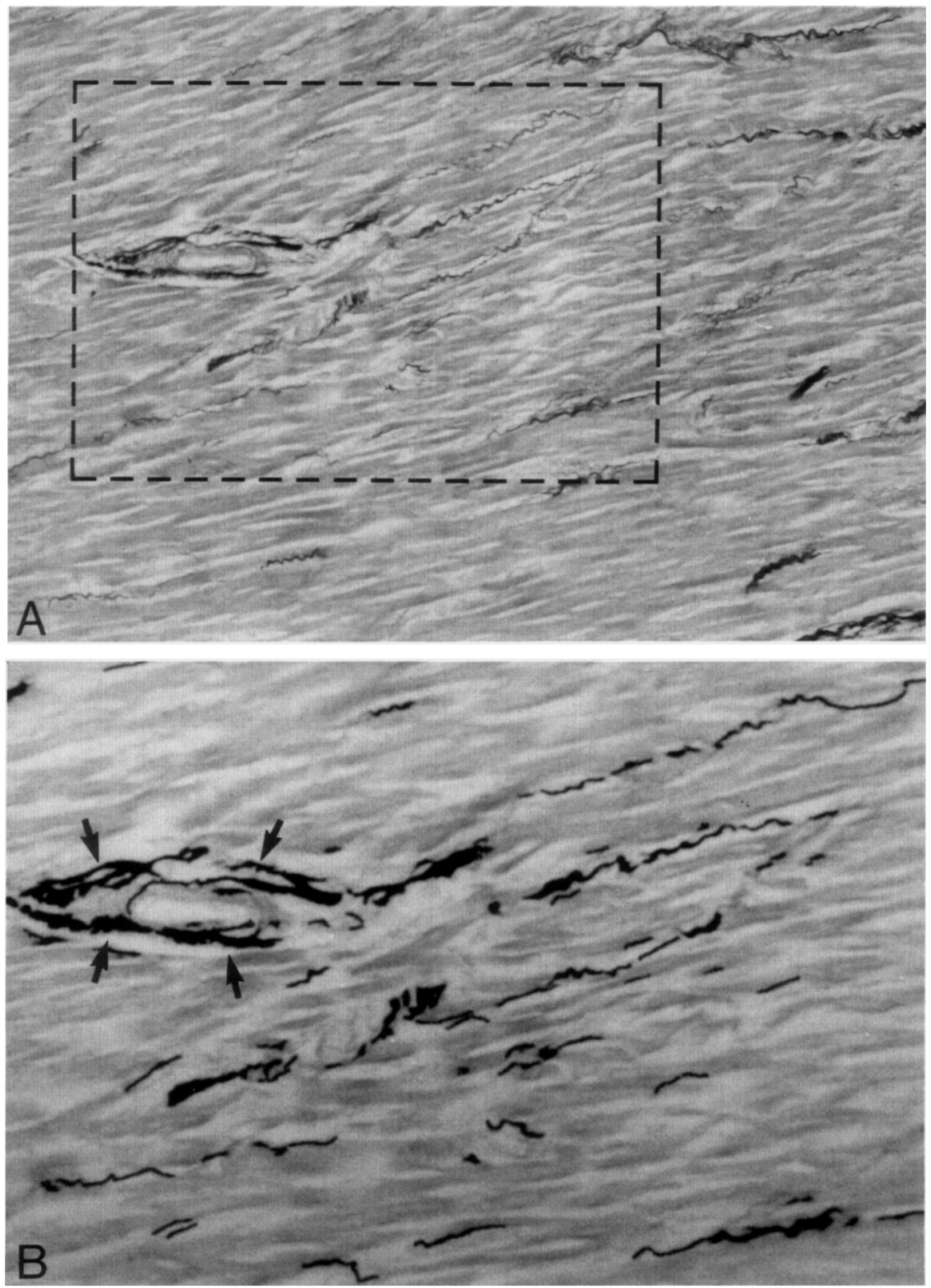

Figure 1. (A) Myocardium stained with Sirius red (original magnification $\times 90$ ). (B) Higher magnification (originally $\times 200$ ) of processed area of the indicated area of panel A. Collagen is black, myocytes are grey, and shrinkage artifacts are white. Perivascular collagen (arrows) is excluded from the analyzed fields. 
tors which might contribute to the development of interstitial myocardial fibrosis.

\section{Methods}

Tissues: normal hearts. Sixteen hearts were provided by the Rotterdam Heart Valve Bank (Bio-Implant Services/Eurotransplant Foundation, Leiden, Netherlands). The patient group was composed of 9 males and 7 females with a mean agc of 26 ycars. Nonc of the paticnts suffered from cardiovascular disorders (trauma capitis, cerebrovascular accident, etc.). The aortic and pulmonary valves had been removed and stored to be used for homografting. All hearts were histologically examined before the aortic and pulmonary valves were used for transplantation. The hearts showed no macroscopical or major light microscopical abnormalities.

Tissues: transplanted hearts. Myocardial tissue from the right and left ventricular free wall and the interventricular septum was sampled during autopsy of 30 patients who died after heart transplantation. The mean interval between transplantation and death was 24 months (range, 6 hours to 91 months). The mean allograft cold-ischemia time was 162 minutes (range, 102-250 minutes).

Clinical data. The mean donor age was 24 years (range, 15-41 years). Death of the donors was caused either by intracranial hemorrhage or acute head injury. The mean age of the recipients at the time of operation was 45 years (range, 16-62 years). Transplantation indications were ischemic heart disease (14 patients), dilated cardiomyopathy (14 patients) and myocarditis ( 2 patients). Six died of acute rejection, 6 of malignancy, 7 of sepsis/infection, 6 of graft arteriosclerosis/ myocardial infarction, 3 of right ventricular failure, 1 of a cerebrovascular accident, and 1 patient died during the transplantation procedure because of rupture of the suture of the aortic anastomosis. HLA-DR and -AB mismatch of the do- nor heart was expressed as the number of mismatched loci. In patients with a short survival time the mean systolic and diastolic blood pressures were determined in the posttransplantation period, whereas mean systolic and diastolic blood pressures of patients with an intermediate and long survival time were taken to be most relevant in the last weeks or months prior to death. A cytomegalovirus (CMV) infection was defined as any appearance of IgM; any isolation of CMV from urine, throat wash, or blood; or any demonstration of the antigen. Acute cellular rejections were treated with a pulsed high dose of methylprednisolone or, in case of refractory rejection, with anti $\mathrm{T}$ cell therapy.

Histological evaluation. In all hearts transmural sections were obtained halfway between the apex and the atrioventricular junction of the right and left ventricular lateral wall and interventricular septum. Sections were fixed in $4 \%$ buffered formaldehyde and paraffin embedded, and 5- $\mu \mathrm{m}$ thick sections were stained with hematoxylin and eosin, elastic-van Gieson, and Sirius red, which specifically stains collagen fibers (21). Graft coronary vasculopathy (concentric or eccentric intimal thickening) was scored semiquantitatively in parafffin sections of the proximal, middle, and distal parts of the right coronary artery, the anterior descending artery, and the circumflex (absent; slight: not exceeding $30 \%$ of the luminal area; moderate: not exceeding $70 \%$ of the luminal area; severe: exceeding $70 \%$ of the luminal area). Graft rejection was scored in routine endomyocardial biopsies by the presence of at least a mild acute rejection according to the Billingham criteria (22).

Morphometry. To quantify the Sirius-red-stained myocardial tissue, we analyzed the histological sections using a Zeiss UEM light microscope (Carl Zeiss, Oberkochen, Germany) coupled to a CCD video camera (Panasonic WV-CD50, Japan) and a digital image analyzer (IBAS with an AT-386 host computer, Kontron Munich, Germany). The microscope
Figure 2. Mean collagen content and standard deviation in the subendocardium $(E)$ and center $(C)$ of the right $(R)$ and left $(\mathrm{L})$ ventricular free wall and interventricular septum $(\mathrm{S} ; \mathrm{RE}, \mathrm{RC}, \mathrm{LE}, \mathrm{LC}, \mathrm{SE}$, and $\mathrm{SC}$, respectively) of normal and transplanted hearts. Bars indicate standard deviation ( $\square$ normal, transplant).

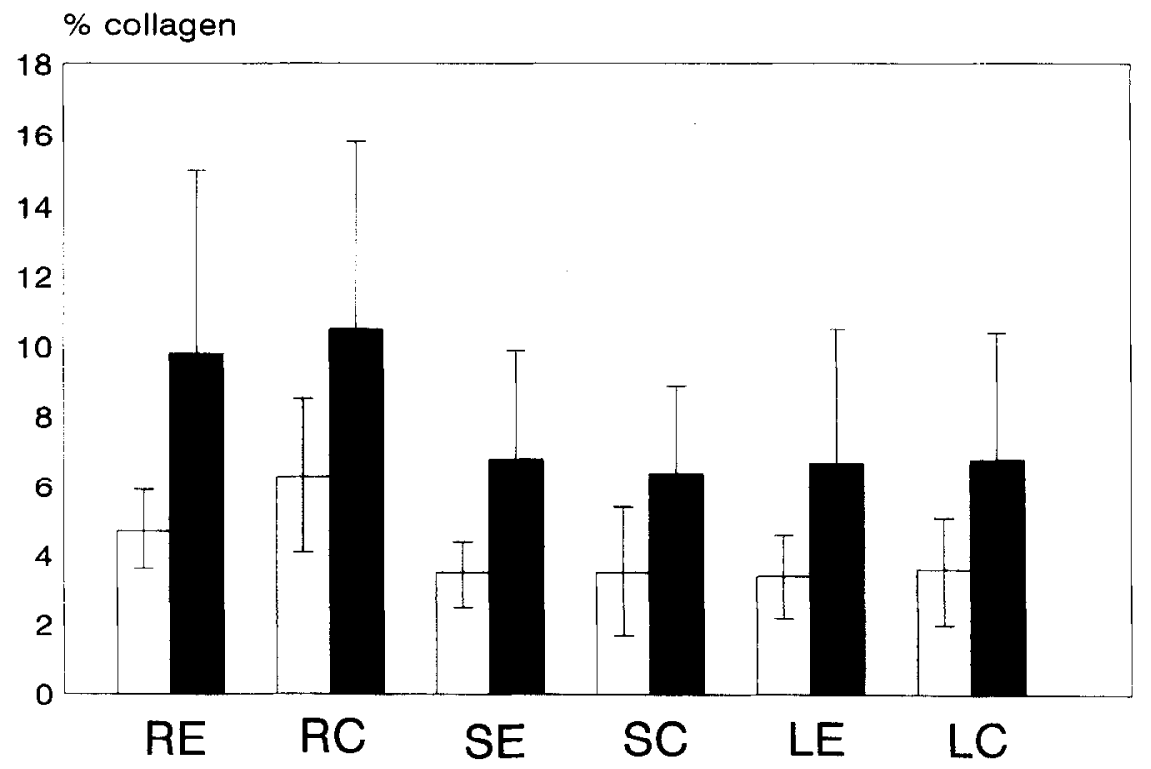



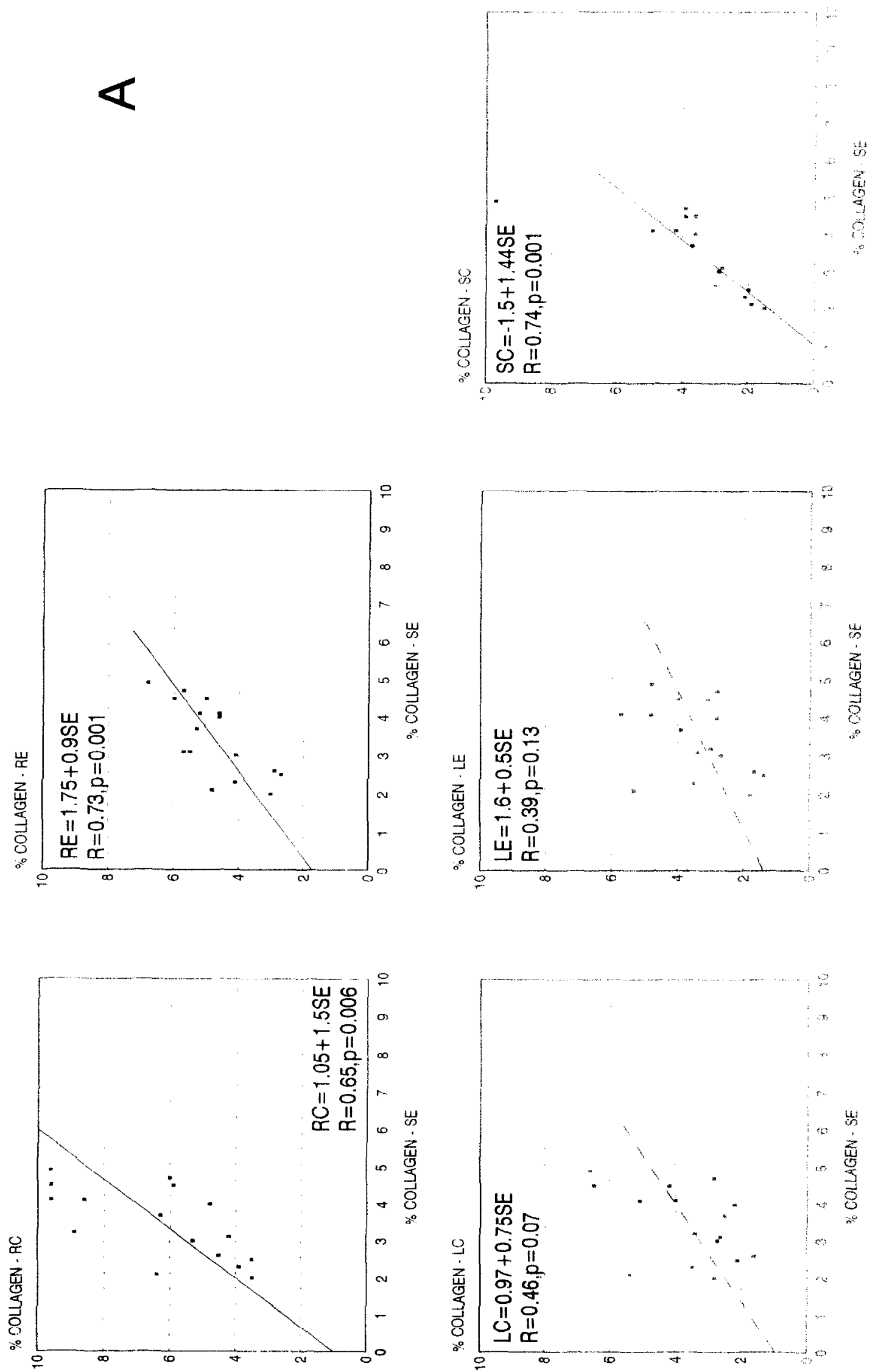

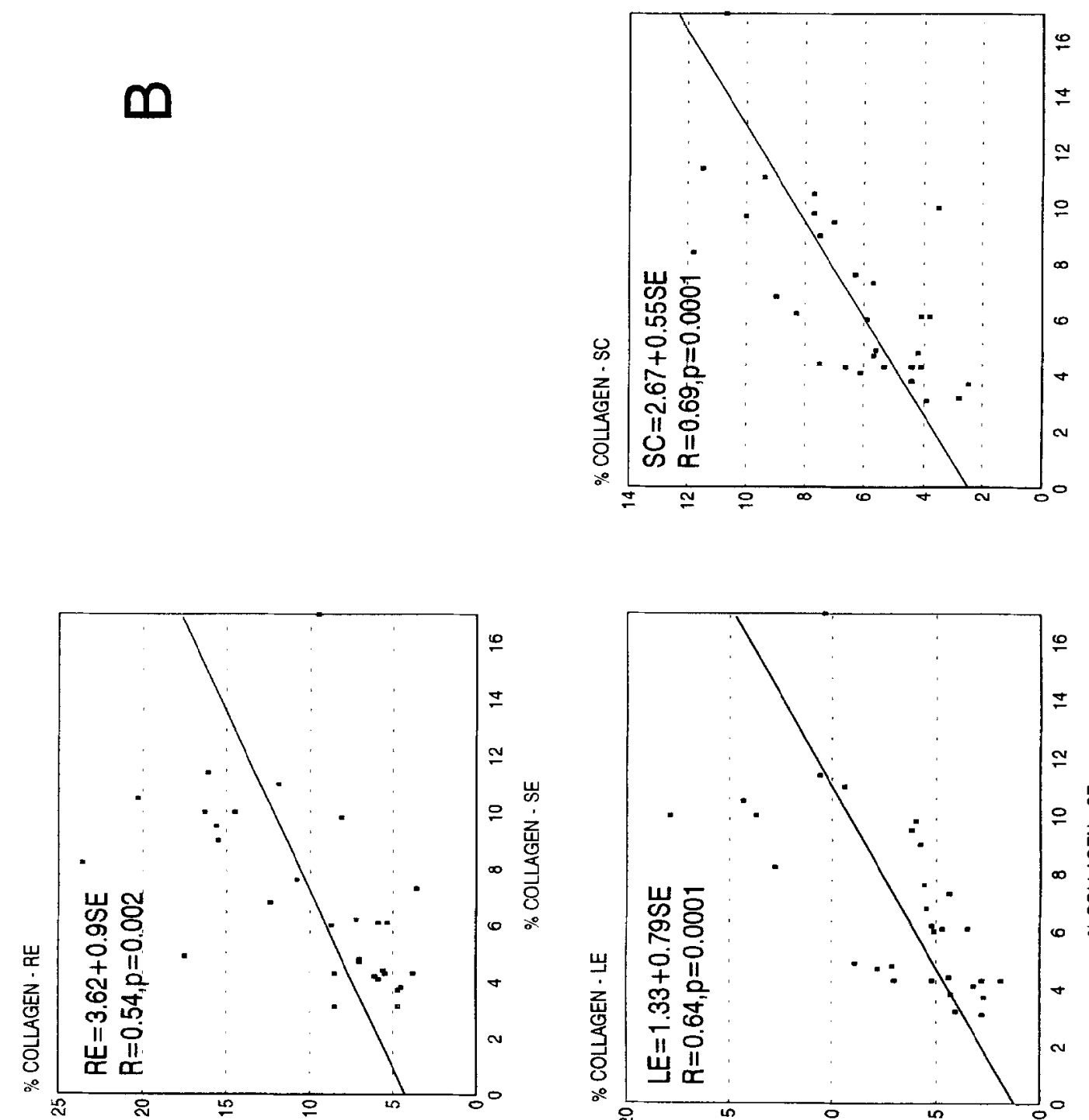

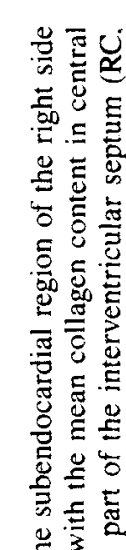

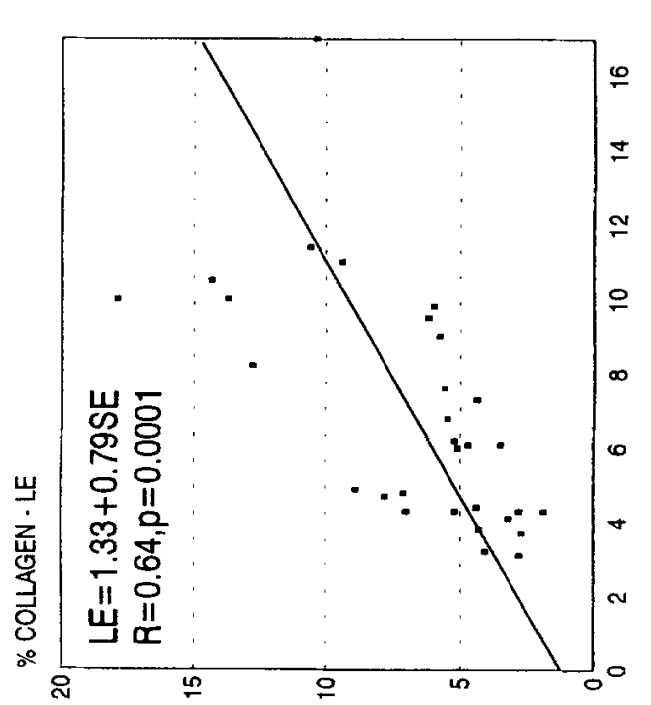

类范

部

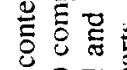

领

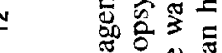

㟧

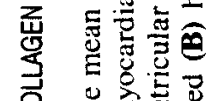

递

एँ

娄到

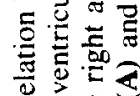

호의

등

के
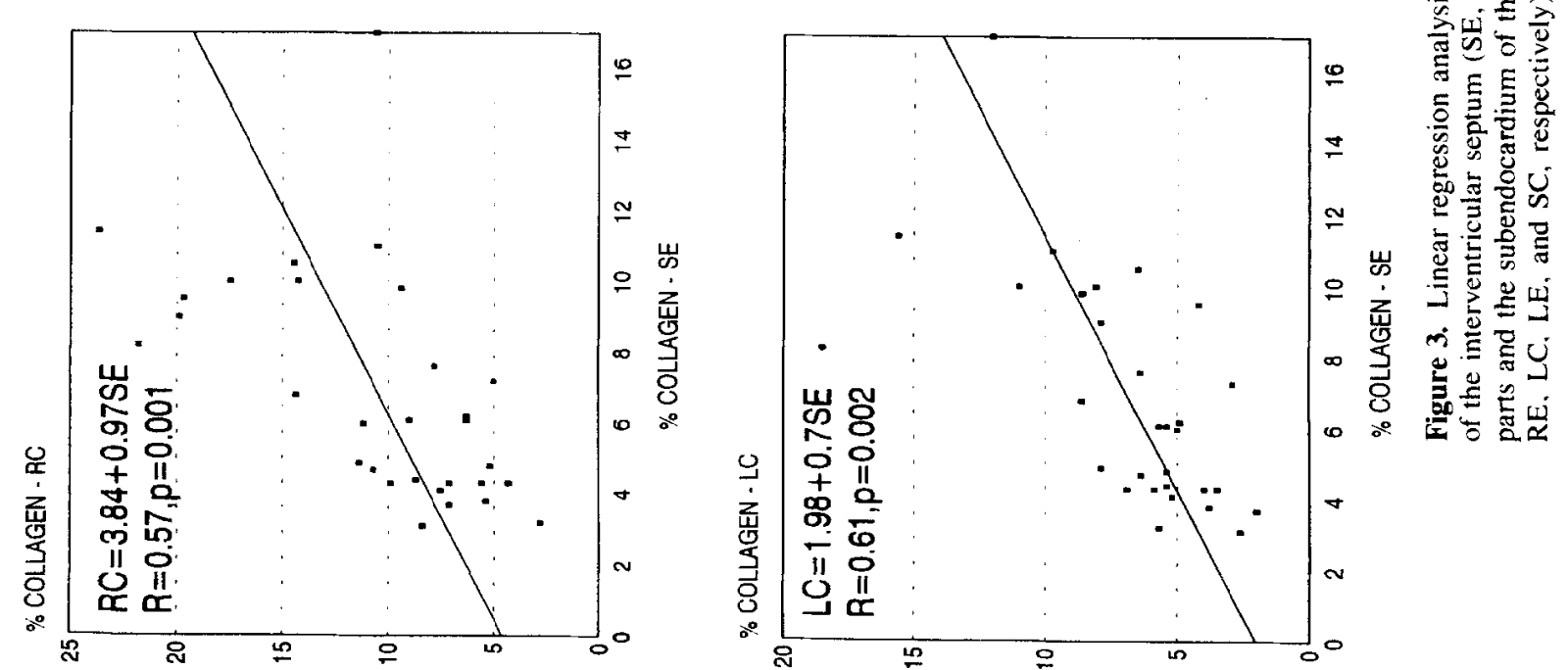
was equipped with a $10 \times$ Planapo objective and a halogen lamp, the voltage of which was controlled by a stabilized power supply (all Carl Zeiss, Oberkochen, Germany).

In each of the transmural sections of the right and left ventricular wall and the interventricular septum, 20 random consecutive adjacent fields (magnification $\times 200$ ) were analyzed directly subendocardial (to a depth of $1 \mathrm{~mm}$, excluding the trabeculations) and in central (halfway between endocardium and epicardium) parts of the section. The central part was selected because pilot experiments indicated this was not significantly different from subepicardial myocardium. The endocardium itself was excluded from the analyzed field, as were blood vessels and perivascular tissue. In order to optimize contrast between collagen, cardiac muscle. and tissue shrinkage artifacts we used a green filter $(551 \mathrm{~nm})$. In each heart the grey level threshold was determined manually, in order to achieve segmentation into three different components: collagen, noncollagenous tissue, and shrinkage artifacts (no tissue; Figure 1). The Sirius-red-stained area was expressed as a percentage of the total measured area of cardiac tissue. The number of measurements was limited to 20 because more measurements did not decrease the coefficient of variance significantly. A number of measurements were repeated in the pilot phase of the project. The intra- and interobserver variation did not exceed $15 \%$.

Statistical analysis. Data were analyzed using an SAS statistics program. Comparisons of the mean collagen scores were made using the paired Student $t$ test. In order to compare the collagen content in the subendocardial region and central parts of the right and left ventricular wall and interventricular septum, we performed linear regression and correlation analysis. The level for statistical significance was chosen at $p<0.05$.

\section{Results}

Normal hearts. The mean collagen content in the subendocardium (RE) and center (RC) of the right ventricle was found to be $2 \%$ higher than that in the interventricular septum (SE and SC) and the free wall of the left ventricle (LE and LC; both $p=0.0001$; Figure 2). In the right ventricle, the collagen content in the subendocardial region was $1.5 \%$ less than that in the center of the myocardium $(p=0.0017)$. No statistically significant differences in collagen content were found between these regions in the interventricular septum and the free wall of the left ventricle. The collagen content in the interventricular septum and left ventricular free wall was almost the same. As indicated by the high standard deviation, ranging from 0.94 in the subendocardial region of the interventricular septum to 2.2 in central parts of the right ventricular wall, there is high interindividual variation in the collagen content between normal hearts.

Figure 3A shows the results of a linear regression analysis of the correlation between the mean collagen content in the subendocardial region of the right side of the interventricular septum (site of right ventricular endomyocardial biopsy) and all other sites. A good correlation was found between the values in the subendocardial region of the right side of the interventricular septum and those in the center of the inter ventricular septum and both regions in the wall of the right ventricle. In contrast, a poor correlation existed with the value: of both regions of the free wall of the left ventricle. No statist cally significant correlation was found hetween donor age and mean collagen content of the hearts.

Transplanted hearts. Overall. the transplanted heart. showed an approximately twofold increase of the mean myocardial collagen content compared with normal hearts f Fig ure 2). In the transplanted hearts the mean collagen contem of the right ventricular wall was $3 \%$ higher than that in the free wall of the left ventricle and interventricular septum. Figure 3B shows that the mean collagen content in the myocas dium at the site of right ventricular endomyocardial biopsy highly correlated with that of the right and left ventricular free wall (subendocardial region and center) and center of the interventricular septum. There is a positive statisucally significant correlation between the mean collagen content of the myocardium and the posttransplantation interval $(R=0.39$ $p=0.03$ : Figure 4). Comparisons of the mean collagen con tent of left and right ventricle and interventricular septum in dividually with the posttransplantation interval shows that the increase in collagen is attributable mainly to an increase in the collagen content of the free wall of the left ventricle $(k$ $0.48, p=0.005$ ). In the first two weeks after transplantation the transplanted hearts showed an increase of $1.4 \%$ of the mean: collagen content compared with normal hearts $(p=0,0$ ) Figure 4).

No statistically significant correlation was found between the collagen content of the myocardium and allograft ische mia time; severity of graft coronary vasculopathy; donor agc: cyclosporin-A treatment: CMV infection: HLA-DR - - a of - B mismatch; or number of rejection episodes. As almost al patients were hypertensive and only insignificant differences in mean systolic and diastolic blood pressure existed between different patients. statistical analysis with regard to the rela tion of the mean collagen content was not possible

\section{Discussion}

Only a few reports have addressed the problem of whether or not small endomyocardial biopsies of normal and transplanted human hearts can be regarded as representative for the entire myocardium. In this study we evaluated whether or not the collagen content in right ventricular endomyocardial biopsies reliably reflects the composition of the entire myo cardium and, therefore, can be used to evaluate to what $\mathrm{ex}$ tent fibrosis affects left ventricular function. The Sirius red morphometry technique allows localization of collagen in transmural sections. Results obtained with the Sirius red technique are comparable to results of an hydroxyproline assay (23) 
Figure 4. Overall mean collagen content of transplanted hearts from patients with short, intermediate, and long survival time compared with the mean collagen content of normal hearts (- mean).

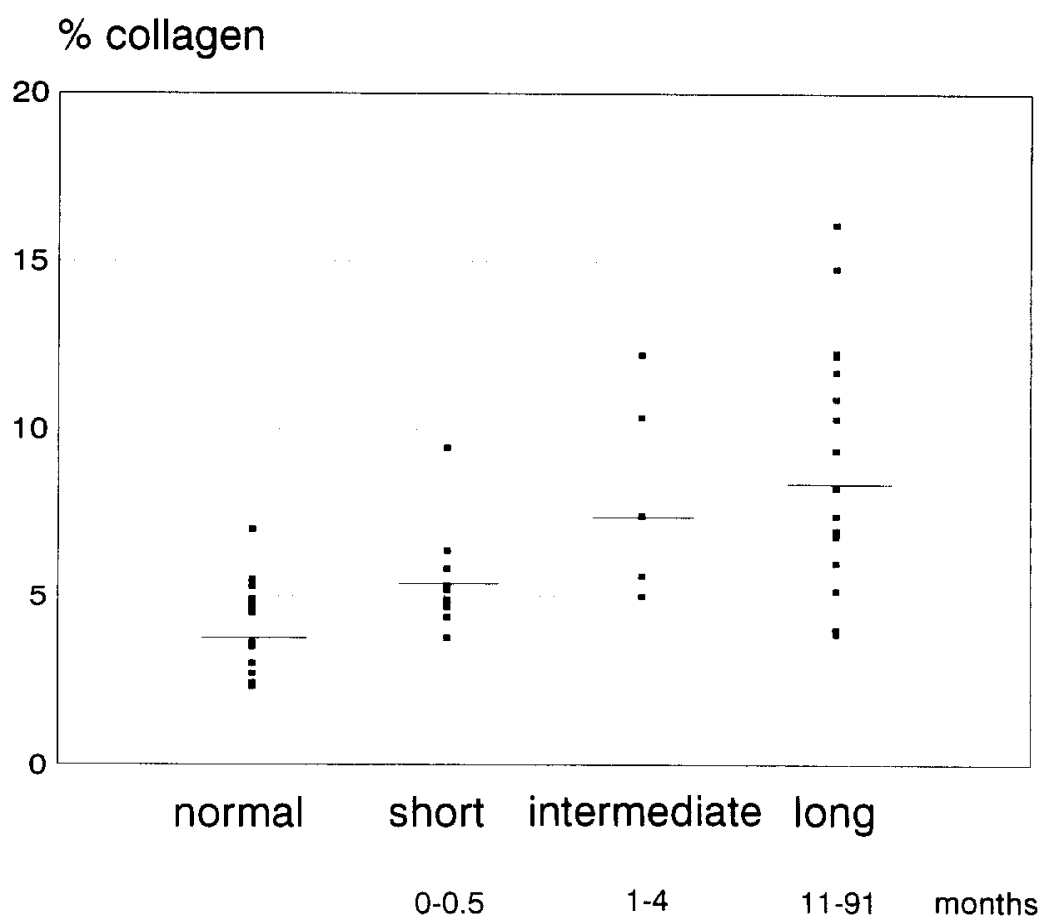

Like Caspari et al. (24) and Oken and Boucek (25), we found, in normal hearts, a significantly higher collagen content $(2 \%)$ in the right ventricular wall than in the left ventricular free wall and interventricular septum.

In normal human hearts the mean collagen content of the subendocardial region of the right side of the ventricular septum correlates well with the mean collagen content of the wall of the right ventricle and the center of the interventricular septum, but it does not reflect reliably the collagen content of the free wall of the left ventricle.

In the transplanted human hearts the mean collagen content of the right ventricular wall was $3 \%$ higher than that in the interventricular septum and the free wall of the left ventricle. The mean collagen content of the right ventricular endomyocardial region in transplants correlates highly with that of the entire myocardium. The reason why this was found only in the transplanted hearts is not clear. The most likely explanation is that interindividual variability obscures the correlation between the different sites in the normal heart. In the transplants, however, diffuse deposition of collagen leads to overall higher collagen content with a high correlation between the different sites. We conclude that, in heart transplant patients, right ventricular endomyocardial biopsies have a potential value in the analysis of the causes of left ventricular dysfunction.

In transplanted human hearts, the posttransplantation survival time correlates positively with the collagen content, and this is attributable mainly to an increase in the collagen of the left ventricular free wall. We consider the left ventricular free wall and interventricular septum as a functional unit and therefore speculate that differences in collagen deposition between the left ventricular free wall and interventricular sep- tum are related to differences in microvasculature and/or hemodynamic load. Further studies will be required to elucidate the mechanisms responsible for these regional differences.

The overall increase in collagen content in the transplanted hearts is attributable to a combination of reparative and reactive fibrosis. We hypothesize that in the first weeks after transplantation, collagen deposition is a result of mainly reparative fibrosis, whereas in the later phase after transplantation reactive fibrosis is a more important factor. All donor hearts were obtained from patients with either intracranial hemorrhage or acute head injury, which are (probably related to intense efferent cardiac sympathetic activity) common causes of myocyte necrosis (26). In some normal hearts focal individual myocyte necrosis was observed, and it is very likely that myocyte necrosis also occurred in donor hearts used for transplantation, though the extent of myocyte necrosis prior to transplantation could not be identified. Necrotic myocytes are replaced by collagen (reparative fibrosis), which becomes identifiable after one week (27). In the normal hearts, not used for transplantation, the interval between intracranial hemorrhage or head injury and explantation of the heart was too short (several hours to three days) to influence the collagen content of the myocardium. However, reparative fibrosis as a result of myocyte necrosis in donor hearts used for transplantation may contribute to an increase in collagen content in patients who survived more than one week. In hearts of patients who died within two weeks after transplantation, there is an increase of $1.4 \%$ in mean collagen content compared with normal hearts. Myocyte necrosis occurred as a result of pre- and perioperative ischemia (28) or is related to intracranial hemorrhage or head injury. In hearts of patients with a intermediate and long survival time (1-91 months), col- 
lagen deposition is a result of a combination of reparative and reactive fibrosis. We were not able to identify individual factors known to play a role in reparative or reactive fibrosis as responsible for the increased collagen content.

We thank M. J. A. P. Daemen and J. P. M. Cleutjens for their stimulating discussions. C. van Krimpen for technical suppont and F. L van der Panne for excellent photography

\section{References}

I. Bishop JE. Greenbaum R, Gibson DG, Yacoub M, Laurent GJ. En hanced deposition of predominantly type 1 collagen in myocardial dis ease. J Mol Cell Cardiol 1990;22:1157 \& 165

2. Thiedemann KU, Holubarsch CH, Medugorac I, Jacob R. Connective tissue content and myocardial stiffness in pressure overload hypertrophy: a combined study of morphologic, morphometric, biochemical and mechanical parameters. Basic Res Cardiol 1983;78:140 155.

3. Jalil JE, Doering CW, Janicki JS, et at Structural vs contractile protein remodeling and myocardial stiffness in hypertrophied rat left ventricle. J Mol Cell Cardiol 1988;20:1179-1187

4. Pfeffer JM. Pfeffer MA. Fishbein MC, Froelich IED. Cardiac function and morphology with aging in spontaneously hypertensive rat. Am J Physiol 1979;6:461 468.

5. Jalil JE, Doering CW, Janicki JS, Pick R, Shrofl SG, Weber KT. Fibrillar collagen and myocardial stiffness in the intact hypertrophied rat left ventricle. Circ Res 1989;64:1041 1050.

6. Weber KT, Brilla CG. Pathological hypertrophy and cardiac interstitium fibrosis and renin-angiotensin-aldosteron system. Circulation 1991: 83 : $1849-1865$.

7. Abrahams C, Janicki JS, Weber KT. Myocardial hypertrophy in macaca fascicularis; structural remodeling of the collagen matrix. Lab Invest $1987 ; 56: 676-683$

8. Weber KT, Brilla C ( 3 , Janicki JS. Myocardial fibrosis: functional significance and regulatory factors. Cardiovasc Res 1993;27:341-348.

9. Weber KT, Pick R, Jalil JE, Janicki JS, Carrol EP. Patterns of myocardial fibrosis. J Mol Cell Cardiol 1989; 21:121-131.

10. Doering CW, Jalil JE, Janicki JS, et al. Collagen network remodeling and diastolic stiffness of the rat left ventricle with pressure overload hypertrophy. Cardiovasc Res 1988;22:686-695.

11. Volders PGA, Willems IEMG, Cleutjens JPM. Arends JW, Havenith MG. Daemen MJAP. Interstitial collagen is increased in the noninfarcted human myocardium after myocardial infarction. $\mathrm{J}$ Mol Cell Cardiol $1993: 25: 1317-1323$.
12. Young JB, Leon CA, Shon D, al avolution ol hemodynamics ate orthotopic heart and heart-lung transplantation: arly restrictive pat terns persisting in occult fashion. I Heart Transplant 1987:6 34.4

I: Human DP. McKenzie FN. Kustuk WJ. Restrictod myoardin am pliance one year following cardiac transplantation. I Heari Iransplan $1984 \cdot 3 \cdot 341 \ldots 345$

14. Rowan RA, Billingham ME Pahologic changes in the fong ieme thin planted heart. Hum Pathol 1900:21,767 772

is Tazelaar HD, Gay RE. Rowan RA, Billingham ME, Gay 5 f Giliget profile in the transplanted heart. Hum Pathol 1090:21:424 420

if Greenberg ML. Ureisky BF, Reddy PS, et al. Long-term hemolynam follow up of cardiac transplant patients treated with cyclosporine and prednisone Circulation 1985:71:487 494

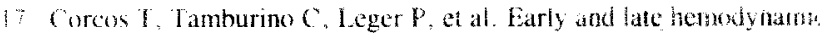
cvaluation after cardiac transplantation: at study of 38 cases. Am $\mathrm{Col}$ Cardio! $1988: 1 ! 264269$

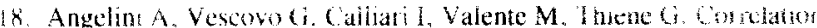
between endomyocardial hiopsies and ventricte tull-thickness sample: in dilated cardiomyopathy: a study of mvocytes and fibrosis ardin asc Pathol 1994: $3 \cdot 167 \ldots 17$

19. Symman WF Nielsen H. Dell R. Rose I: Marbot Ce Candac at lograft pathology: A clinicopathologic correlaton. Cardiovase samo $1994: 2 \cdot 249 \ldots 256$

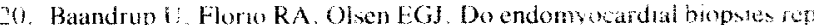
resent the morphology of the rest of the myocardium? A quantitative light microscopic study of single $\mathrm{v}$. multiple biopsies with the King: boptone. Eut Heart J 1982:3:17! - 178

21. Junquera LCL: Bicnolas G, Brentani RR. Picosirius staining plus pola isation microscopy: a specific method for collagen detection in tissu sections, Histochem J 1979;11:447-455.

22. Billingham ME. Diagnosis of cardiac rejection by andonyocandial bi opsy. Heart Transplant 1982:1:25-30

23. Dolber PC. Spach MS. Picosirius red staining of sardiac muscic fol lowing phosphomolybdic acid treatment. Stain Technol 1987,62:23-26

24. Caspari PG, Newcomb M, Gibson K, Harris P. Collagen in the nomat and hypertrophier human ventricle Cardiovas: Res 1977:11:554-558.

25. Oken DL. Boucek RJ. Quantitation of collagen in human myocardium Circ Res 1957:5:357 361

66. Reichenbach DD, Benditt EP. Catecholamines and cardiomyopathy Hum Pathol 1970; $1: 125$ 150

27. Weber K'T. Brilla CG. Factors associated with reactive and repan tive fibrosis of the myocardium. Basic Res Cardiol 1992;87(Suppl 1) $291 \ldots 301$

28 Smith JA Mottram PL, Mirisklavos A Mason A, Dumble LJ. Ciunic GJA. The effect of operative ischemia in murine cardiac transplantation: isograft control studies. Surgery 1987:101:86-90 